\title{
A Robotic System to Locate Hazardous Chemical Leaks.
}

\author{
R. Andrew Russell \\ Intelligent Robotics Research \\ Centre \\ Department of Electrical \& \\ Computer Systems Engineering \\ Monash University, Clayton, \\ VIC 3168, AUSTRALIA
}

\author{
David Thiel and Reimundo Deveza \\ School of Microelectronic \\ Engineering \\ Griffith University, Nathan, \\ Brisbane, QLD 4111, \\ AUSTRALIA
}

\author{
Alan Mackay-Sim \\ School of Science \\ Griffith University, Nathan, \\ Brisbane,QLD 4111, \\ AUSTRALIA
}

\begin{abstract}
Tracing leaks of airborne radio-active, poisonous or flammable materials is a potentially dangerous activity that could be undertaken by robotic systems. This paper describes a practical investigation of the possibility of developing simple, low cost, autonomous robots to perform this task. It is envisaged that these robots would be used in large numbers. This would improve reliability through redundancy and provide large area coverage from a wide distribution of robots. Failure of a single unit would not jeopardise the overall sensing operation. Sensing and control techniques developed to perform leak location in a simplified laboratory environment are described and experimental results are presented.
\end{abstract}

\section{Introduction}

The search for hazardous chemical leaks is an ideal application for robotic technology and has been suggested in general terms by several authors $[1,2]$. Being dispensable, robotic devices could be sent into the most dangerous situations. A robotic leak detector could be engineered to survive extreme conditions of radiation and/or toxic chemical concentration. To protect a human investigator from these extremes would be much more difficult.

The project described in this paper examined the basic requirements for a robotic system capable of performing chemical leak location. For this preliminary investigation the environment was simplified to a flat area with discrete, widely spaced obstacles, constant wind intensity and unvarying wind direction. To provide manoeuvrability the mobile robot consisted of a small 'turtle geometry' robot vehicle. This vehicle was equipped with a collision sensing bumper, a chemical sensor and a wind vane. Using this equipment and experimental environment, control algorithms were developed to perform the leak location task.

The use of multiple cooperating robots has been proposed for the task of locating the source of volatile chemical leaks [3]. In this project the robots are intended to be self contained (except where they report their findings to some central location) and act with complete autonomy. Multiplicity is seen as a way of insuring against failure of an individual system and to provide wide coverage [4]. An artificial sense of smell for locating the source of volatile chemicals has been suggested by Rozas, et al. [5]. However, their paper mainly deals with odour discrimination while techniques for tracing and identifying the source are not considered in detail.

\section{The mobile robot platform}

Major elements of the leak locating system are shown in Figure 1. The mechanical configuration of the mobile robot is based on 'turtle' geometry with two side-by-side wheels balanced by a captive ball bearing to give a third point of contact with the ground. Each wheel is driven by an ironless rotor dc motor through a 40:1 gearbox.. An Intel 8035 microcontroller controls wheel motion using feedback from optical encoder disks incorporated into each wheel. This microcontroller also monitors a wind vane to determine wind direction relative to the robot and registers collisions with obstacles using a bumper ring surrounding the robot.

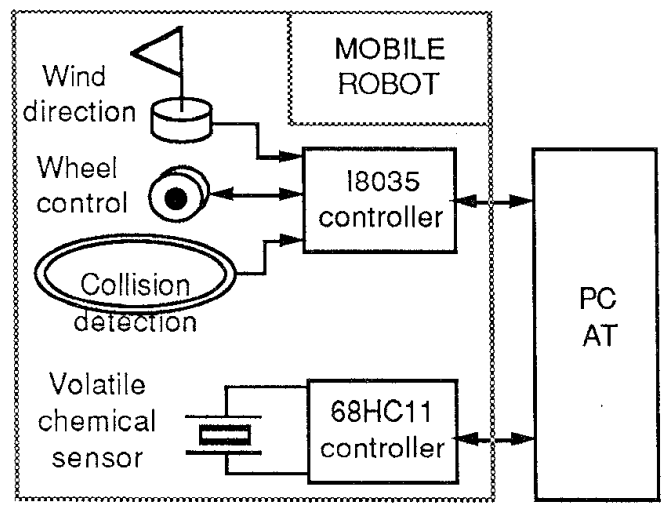

Figure 1. A block diagram of the complete leak locating system.

The leak location algorithms developed in this project require information about the position of the robot relative to its location at earlier times. On the smooth floor used in our experiments odometry was sufficiently accurate. In practice other techniques may be more appropriate such as 
GPS (global positioning by satellite) or the use of fixed beacons. For this application of locating the source of chemical leaks the most important sensor is the one that measures the concentration of volatile chemicals.

\subsection{Sensing volatile chemicals.}

There are many sensing techniques which could be used for detecting the concentration of volatile chemicals. The quartz crystal microbalance was selected for this application because it rapidly tracks changing chemical concentrations (responding in under one second). This kind of sensor is simple to construct, draws little power and is quite robust. The sensor uses a quartz crystal as a sensitive balance to weigh the odour molecules [6]. A chemical coating on the crystal is chosen to have a specific affinity for the target odourant molecules. When air containing molecules of the target odour passes over the crystal some of the molecules become temporarily attached to the coating. This increases the mass of the crystal and lowers its resonant frequency [7]. A sensitivity of $1 \mathrm{~Hz}$ change in frequency for each part per million of odourant has been measured for this type of sensor [8]. A cross-section view of the sensor crystal is shown in Figure 2.

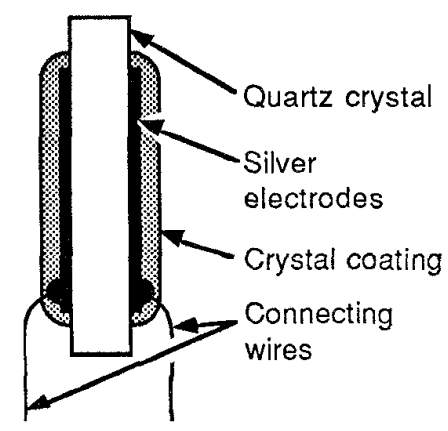

Figure 2. A cross-section view of the quartz crystal sensor.

The volatile chemical sensor employs two crystal oscillator circuits. One oscillator uses a coated crystal which responds to volatile chemical concentration and the other an uncoated crystal to act as a reference. The output of the reference oscillator is subtracted from the sensor oscillator output to provide a difference frequency proportional to chemical concentration. This frequency is measured by a $68 \mathrm{HC} 11$ microcontroller and transmitted in serial form to the PC-AT computer controlling the mobile robot.

In these experiments camphor was chosen for the volatile chemical. It is safe to handle, non-offensive, easily obtained and inexpensive. To detect camphor the quartz crystal sensor was coated with Silicone OV-17. Because of large airflow and relatively low volatility of camphor a large area target was constructed consisting of 14 short sections of cardboard mailing tube glued together.
A solution of camphor in alcohol was sprayed onto the cardboard immediately before each experiment.

\section{Control of the robot.}

The program controlling the overall search strategy ran on the PC AT personal computer. Sensors and the robot vehicle were controlled and interrogated via two RS232 serial ports. The software was written in Turbo Pascal but it was structured in the form of production rules for portability and to facilitate debugging.

Phase 1: plume acquisition.

For safety of its human operators a robot would be released at a point adjacent to a chemical plume rather than actually in it. The robot tracks away from the release point in a cross-wind direction monitoring chemical concentration (Figure 3). Upon detecting the edge of the plume the robot starts recording sensor readings as it moves to establish the distribution of chemical concentration in a cross-section of the plume. When the robot reaches the far side of the plume it stops and calculates the centroid of the plume cross-section. The robot then returns to the calculated centroid position, turns into the wind and follows the airborne chemical towards its source.

Rules controlling plume acquisition:

Initially: flag $=$ false and the robot moves to orientate itself across wind.

(Rule 1) If (flag $=$ false) then (move small distance $e$ )

(Rule 2) if (flag $=$ false) and (chemical detected) then (flag $=$ true)

(Rule 3) if (flag = true) and (chemical detected) then (move small distance $\mathrm{e}$ )

(Rule 4) if (flag = true) and (no chemical detected) then (finish)

In our experiments $e$ was chosen to be $35.5 \mathrm{~mm}$.

During phase 1 chemical intensity readings are taken at regular intervals as the robot moves at right angles to the wind. Sample $n$ is taken at a distance $d_{n}$ from the initial position and the measured chemical concentration $t_{n}$ is recorded. A threshold is set to eliminate intrinsic fluctuations in the sensor output.

$$
\begin{array}{ll}
\tau_{n}=t_{n} & \text { if } t n \geq \text { threshold } \\
\tau_{n}=0 & \text { if } t n<\text { threshold }
\end{array}
$$




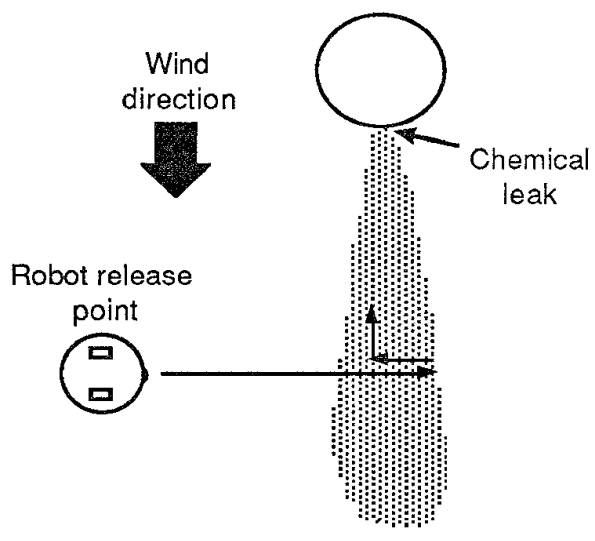

Figure 3. Locating the centre of a chemical plume.

Moments of the distribution are then calculated:

$$
\begin{aligned}
& m_{0}=\sum_{n=1}^{k} t_{n} \\
& m_{1}=\sum_{n=1}^{k} t_{n} \cdot d_{n}
\end{aligned}
$$

The displacement for the initial position of the centroid of the chemical plume $d_{x}$ is given by:

$$
d_{x}=\frac{m_{1}}{m_{0}}
$$

Phase 2: following odour plume.

Turbulence in the airflow produces a fluctuating chemical concentration at the robot. In order to maintain a heading close to the centre of the chemical plume the robot employs a hill-climbing strategy in which the robot turns to take sensor readings to the left and right of its current heading. The heading is then modified so that the robot moves towards the stronger reading. Using a single sensor eliminates problems of matching the response of two separate sensors. After turning the robot travels forwards thirty $3.5 \mathrm{~mm}$ increments.

\section{Phase 3: circumnavigating obstacles.}

During each linear movement the robot monitors its bump sensor to detect collisions with obstacles. When a collision is detected the robot works its way around keeping the obstacle on its right hand side (Figure 4).

In practice the method of tracking round an obstacle will depend on the nature of the information provided by the sensors and the geometry of the robot. In this case low level control of the robot only allows movement in a straight line and turning on the spot. The bump sensor provides contact information.

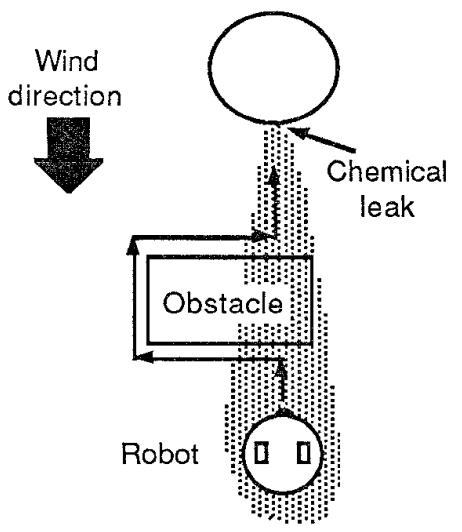

Figure 4. Robot navigates around an obstacle lying in the path of the chemical plume.

Based on these characteristics of the robot and sensor a simple edge tracing strategy was developed which finds points of contact along the edge of the obstacle and always moves the robot in one of four orthogonal directions (this makes the odometry calculations much simpler). Figure 5 shows an example of the path generated by this algorithm. It is assumed that the wind is blowing from the North.

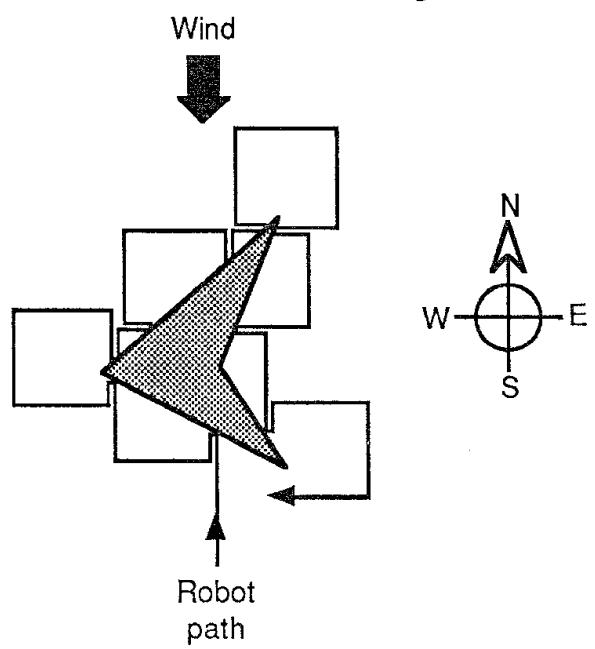

Figure 5 . The path taken by the robot as it tracks round an obstacle.

Rules used to control the edge tracking behaviour [7]:

Initially: flag=false and it is assumed that the robot has just hit an obstacle while moving North

(rule 1) If (moved North) and (hit obstacle) then (backup South) 
(rule 2) If (moved North) and (missed obstacle) then (move East)

(rule 3) If (backedup North) then (move East)

(rule 4) If (moved East) and (hit obstacle) then (backup West)

(rule 5) If (moved East) and (missed obstacle) then (move South)

(rule 6) If (backedup East) then (move South)

(rule 7) If (moved South) and (hit obstacle) then (backup North and set flag=true)

(rule 8) If (moved South) and (missed obstacle) then (move West)

(rule 9) If (backedup South) then (move West)

(rule 10) If (moved West) and (hit obstacle) then (backup East)

(rule 11) If (moved West) and (missed obstacle) then (move North)

(rule 12) If (backedup West) then (move North)

(rule 13) If (chemical detected) and (up wind of obstacle) then (obstacle negotiated)

(rule 14) If (chemical detected) and (near starting point) and (flag=true) then (source located)

(rule 15) If (near starting point) and (no chemical detected) and (flag=true) then (error)

Phase 4: terminating conditions.

In the final phase of the search the robot must determine when it has arrived at the source of the leak. There are several terminating conditions which have been incorporated into the control program. The robot may not always bump into the ruptured tank or pipe that is the source of the chemical leak. If the leak is located above the robot then contact with the volatile chemical will be lost as the robot moves beneath the leak. This situation is illustrated in Figure 6.

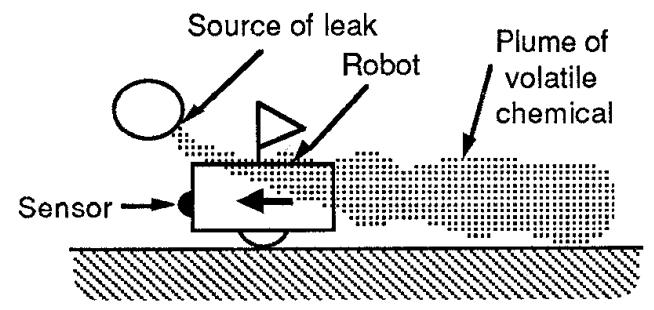

Figure 6. The robot loses track of the volatile chemical as it moves beneath the source.

In a similar way if the volatile chemical is issuing from the ground due to a broken underground pipe then the robot will lose contact as it runs over the location of the leak. Fig. 7 shows this possibility.

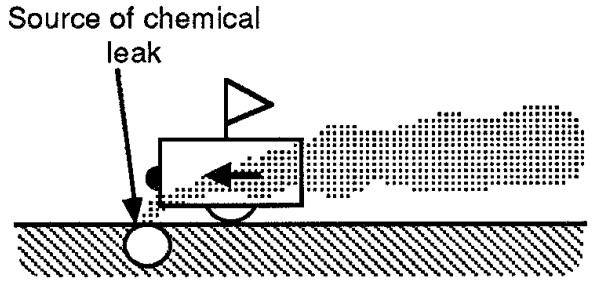

Figure 7. The robot loses track of the volatile chemical as it moves over the source.

For both of the above situations the robot will broadcast the position where it loses the plume as the nearest estimate to the leak location.

However, the most interesting case is where the source is also an obstacle. In this case the robot must track completely around the obstacle to ensure that the volatile chemical originates inside the obstacle and not beyond it. This manoeuvre is illustrated in Figure 8.

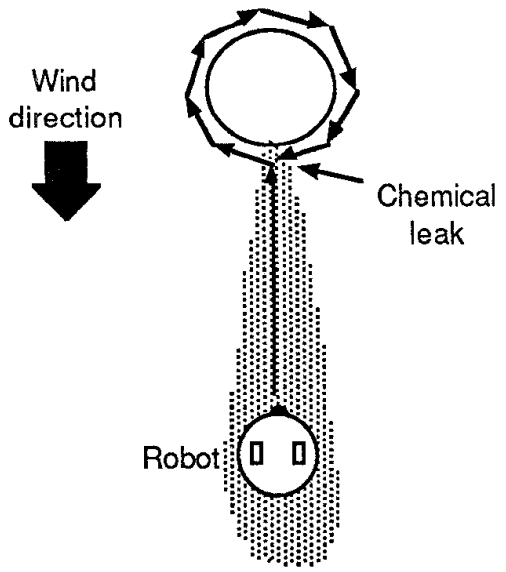

Figure 8. Robot tracks around the source of a chemical leak to ensure that the volatile chemical plume does not originate further up wind.

\section{Experimental results}

Experiments were conducted in a corridor where the airflow was a reasonably constant $0.5 \mathrm{~m} / \mathrm{sec}$. measured $1.5 \mathrm{~m}$ from the floor and less than $0.3 \mathrm{~m} / \mathrm{sec}$. measured $0.2 \mathrm{~m}$ from the floor. The corridor is a public thoroughfare in the Department of Electrical and Computer Systems Engineering at Monash University. Throughout the experiments people walked past adding extra turbulence and disturbance to the airflow. Obstacles were made from blocks of wood held in place by additional weights.

As a first test of the system the robot moved at right angles to the airflow two metres downwind of the chemical source. At each of 31 locations spaced $35.5 \mathrm{~mm}$ apart the chemical concentration was measured. Figure 9 
shows a typical graph of chemical sensor output plotted against robot position.

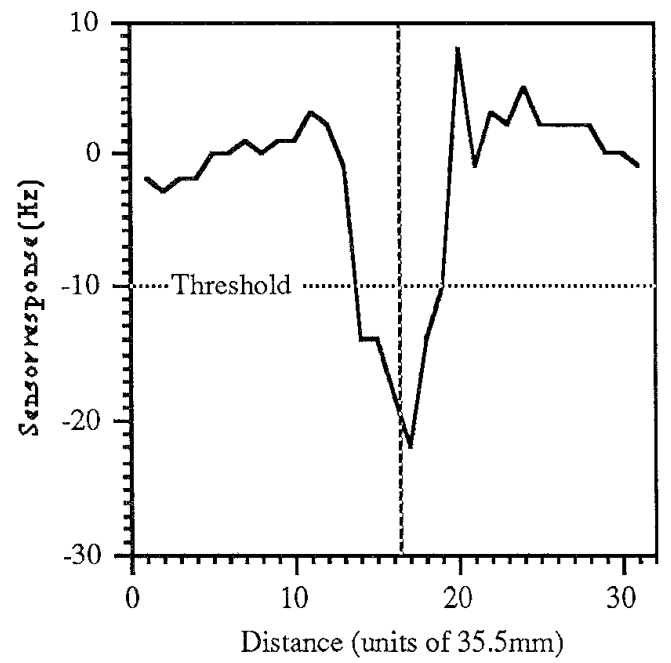

Figure 9 Chemical concentration measurements taken across the chemical plume.

This result demonstrated that the plume of camphor could be detected at a distance and that the first phase worked correctly. Further elements of the control strategy were then tested. The chemical source was placed on a block of wood $400 \mathrm{~mm}$ long and $260 \mathrm{~mm}$ wide. Figure 10 shows the track taken by the centre of the robot as indicated by recorded odometry information. The robot has a $135 \mathrm{~mm}$ radius and this is the reason that the recorded tracks do not touch the obstacle.

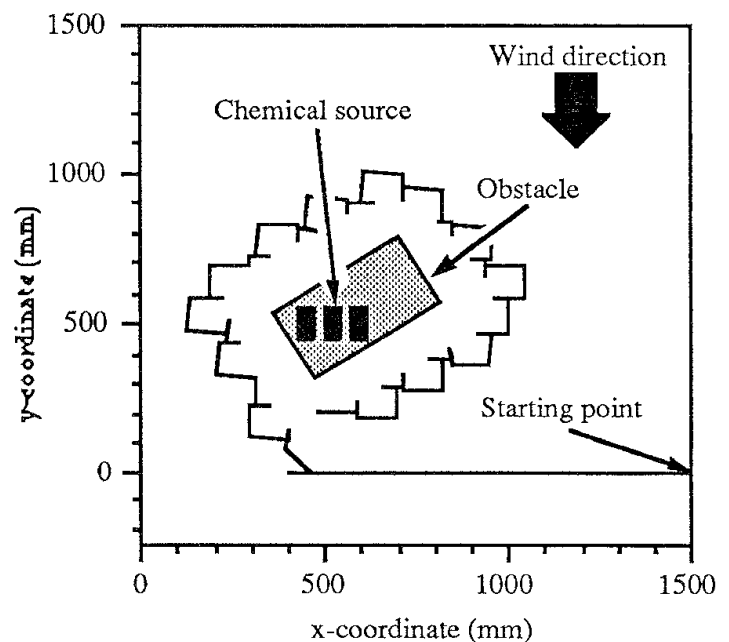

Figure 10 Verifying the source of a chemical leak by circumnavigating the source.
In Figure 10 airflow travels in the negative $y$ direction and the robot starts by moving in the negative $\mathrm{x}$ direction to locate the plume of camphor. Having completed the scan across the airflow the robot returns to the centre of the chemical plume and follows the chemical up-wind. Shortly afterwards the robot collides with the obstacle. It then tracks round and fails to detect the chemical plume on the up-wind side of the obstacle. Upon arriving back near the point of initial contact the robot halts and reports that the obstacle contains the chemical leak.

The final experiment involved introducing an obstacle into the path of the robot. Figure 11 shows that the robot contacts the additional obstacle and tracks around it. When the chemical plume is detected on the up-wind side of the obstacle the robot reverts to following the chemical plume until it meets the obstacle containing the source of the chemical plume. The robot tracks around this obstacle and then reports that it has located the source of the chemical plume.

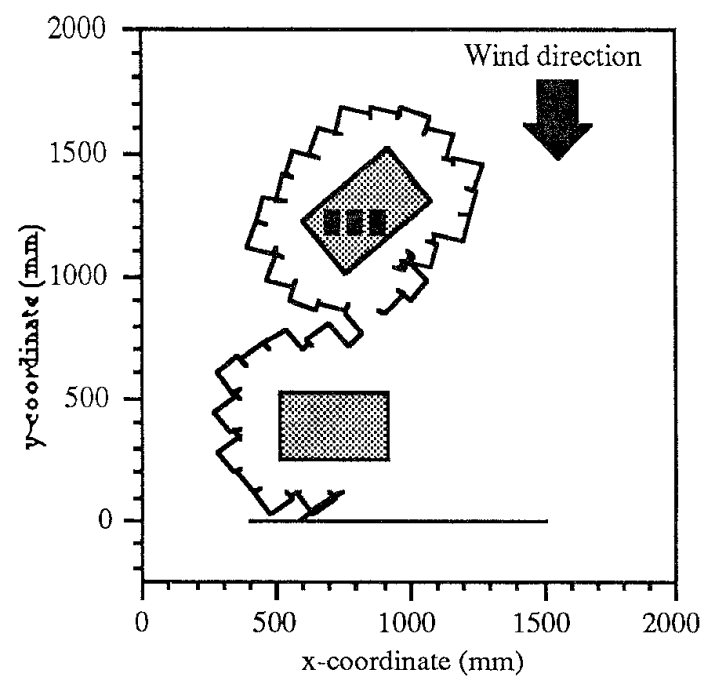

Figure 11. Avoiding an obstacle in the chemical plume.

\section{Conclusions}

The detection and location of dangerous chemical leaks is an ideal application of robotic technology. To demonstrate the feasibility of this approach we have considered a simplified situation with well defined obstacles and reasonably steady airflow. Robot control algorithms have been developed for locating the centre of a chemical plume, following the plume up-wind, recognising and avoiding obstacles and recognising the source of a chemical leak. These algorithms have been successfully tested using a small 'indoor' type robot and utilising the airflow produced by the building air conditioning system to simulate wind blowing steadily from one direction.

In practice the direction and strength of the wind could change and the robot might have to negotiate irregular terrain. Thus, there is plenty of scope for 
improving the agility of the robot platform and incorporating additional sensors and 'intelligence' to cope with the real world. However, this project has demonstrated that the basic ideas are sound and could serve as the basis for developing a practical leak locating robot system.

\section{Acknowledgments}

The authors gratefully acknowledge the assistance of Dr. Don Clegg, Griffith University for advice on chemistry. Reimundo Deveza was supported by a grant from the Australian International Development Assistance Bureau (AIDAB). This project is supported by a grant from the Australian Research Council (ARC).

\section{References}

1. Engelberger, J.F., Robotics in Service, Kogan Page, London, 1989.

2. Larcombe, M.H.E. and Helsall, J.R., Robotics in Nuclear Engineering, Published by Graham \& Trotman Ltd., for the Commission of the European Communities, 1984
3. Genovese, V., et. al.., 'Self organising behaviour and swarm intelligence in a pack of mobile miniature robots in search of pollutants', Proceedings of the IEEE/RSJ International Conference on Intelligent Robots and Systems, Raleigh, July 7-10, 1992, pp. 1575-1582.

4. Flam, F., 'Swarms of mini-robots set to take on Mars terrain', Science, Vol 257, September 18th, 1992, p. 1621.

5. Rozas, R., Morales, J. and Vega, D., 'Artificial smell detection for robotic navigation', Proceedings of the Fifth International Conference on Advanced Robotics, 1991, pp. 1730-1733.

6. Sauerbrey, G.Z. 'The use of quartz crystal oscillators for weighing thin layers and for microweighing',. Zeitscrift fuer Physic, Vol. 155, 1959, pp. 206-222.

7. King, W.H. 'Piezoelectric sorption detector', Anal. Chem., Vol. 36, No. 9, 1964, pp. 1735-1739.

8. Deveza, R., Thiel, D., Russell, R.A. and MackaySim, A. 'Odour sensing for robot guidance', The International Journal of Robotics Research, Vol. 13, No. 3, June 1994, pp. 232-239.

9. Russell, R.A., 'Object recognition using articulated whisker probes', Proceedings of the 15th International Symposium on Industrial Robots, Tokyo, Japan, 1985, pp. 605-612. 